\title{
Dosimetric Evaluation of Deep Inspiration Breath-Hold Technique for Breast Cancer Radiotherapy: A Single-Center Experience
}

\author{
(1) Öztun TEMELLi, (1) Mehmet DEMIRTAŞ, (1) Berat Tuğrul UĞURLU, (1) Eda KAYA PEPELE, \\ (1) Bahaddin YAPRAK, (1) Hülya GÜLBAŞ, (1) Fatma Aysun ERASLAN, (1) Şimay GÜROCAK
}

Department of Radiation Oncology, İnönü University, Malatya-Turkey

\begin{abstract}
OBJECTIVE
To retrospectively evaluate the absorbed doses of organs at risk, such as lungs and the heart of the patients who underwent radiotherapy (RT) using the voluntary deep inspiration breath-hold (vDIBH) technique, and to compare the results with the literature.
\end{abstract}

\section{METHODS}

In this study, 102 patients who underwent adjuvant RT for the treatment of breast cancer in our clinic between November 2018 and December 2019 were included. A breast and/or chest wall, and/or lymph node RT of $50 \mathrm{~Gy}$ in 25 fractions was planned for all patients, and an additional boost of $10 \mathrm{~Gy}$ in five fractions was planned for patients requiring an RT boost. The treatment plans included 3DCRT, Field in Field (FIF), IMRT and VMAT approaches.

\section{RESULTS}

In the group undergoing supraclavicular fossa RT, the mean V20Gy was found to be $24.8 \%$, compared to $16 \%$ in the group not undergoing supraclavicular fossa $\mathrm{RT}(\mathrm{p}<0.01)$. In the group undergoing mammaria interna RT, the mean heart dose was $3.1 \mathrm{~Gy}$, compared to $2.1 \mathrm{~Gy}$ in the group not undergoing mammaria interna RT ( $\mathrm{p}=0.04)$.

\section{CONCLUSION}

Respiratory motion control techniques can reduce uncertainties in the target related to respiratory motion. The irradiated volume doses of the ipsilateral lung, heart reduce.

Keywords: Breast cancer; deep breath hold technique; radiotherapy.

Copyright (๑) 2020, Turkish Society for Radiation Oncology

\section{Introduction}

Breast cancer is the most common cancer type and the leading cause of death among women worldwide.[1] Treatment modalities include surgery, chemotherapy, hormone therapy and radiotherapy (RT). Adjuvant RT aims to eradicate tumor deposits either through mastectomy or breast-conserving surgery and has improved locoregional recurrence (LRR), breast cancer-specific survival and overall survival (OS).[2] Radiation-related toxicity is still a significant problem due to the proximity to at-risk organs, despite the use of modern techniques in RT applications. Various studies have reported an increase in cardiovascular mortality in patients undergoing breast and/or chest wall irradiation. $[3,4]$ It has been shown that breast RT increases 
the risk of secondary lung cancer development, pneumonia and lung fibrosis.[5]

The deep inspiration breath-hold (DIBH) technique has been developed to reduce doses of RT on the lung and heart. There are different methods and systems for the application of DIBH, including voluntary, moderate or active breathing control $[\mathrm{ABC}]$. Voluntary DIBH (vDIBH) relies on patient coaching throughout the respiratory cycle, whereas ABC DIBH makes use of a spirometer-like device. Although both techniques are effective, vDIBH is reported to be better tolerated.[6]

The vDIBH technique is based on observation of the flattening of the diaphragm and the expansion of the lungs during inspiration when the heart is moved away from the chest wall. During both simulation and treatment, the patient takes a deep breath and then holds it for the duration of the treatment. [7] In two extensive studies, a relative dose reduction was recorded in the heart from $40-48 \%$, along with a mean dose reduction of $1.7-$ 2.5 Gy with DIBH when compared to the free-breathing technique.[8,9] Ipsilateral lung doses were also found to be lower with DIBH in patients undergoing additional internal mammary chain (IMC) RT.[10]

The present study to retrospectively analyze the doses of organs at risk, such as the lung and heart of patients who underwent RT using the $\mathrm{VDIBH}$ technique, and to compare them with the findings in the literature.

\section{Materials and Methods}

\section{Patient Selection}

In this study, 102 patients who underwent adjuvant RT for the treatment of breast cancer in our clinic between November 2018 and December 2019 were evaluated retrospectively. The inclusion criteria were no hearing problems, no known respiratory disease, able to perform the given commands and an ECOG performance score of $0-1$. The exclusion criteria were unable to perform the breath-hold technique, bilateral breast cancer, patients undergoing palliative breast RT and those treated with hypofractionated RT. This study was approved by the local ethics committee and was carried out in accordance with the Helsinki Declaration. Patient data is provided in Table 1.

\section{Simulation, Treatment Planning and Delivery}

The breath-hold technique was described to all patients by a training nurse before the simulation. All patients were placed in the supine position on the breast board. The surgical scar and palpable breast tissue were marked with radiopaque wire. A Varian RPM (real-time po- sition management) system (Varian Medical Systems, Palo Alto, CA, USA) was used for the vDIBH technique. The RPM box, placed on the patient's chest wall, detects vertical displacements during the respiratory cycle, and the patient is instructed to hold their breath during a computer tomography (CT) (Aqullion LB TSX-201A, Toshiba Medical Systems, Japan) scan. A specific cutoff value for the upper and lower values of vertical displacement of the patient's chest wall was determined. A CT scan at $3 \mathrm{~cm}$ thickness was performed from the level of the larynx to the center of the abdomen.

Target volume contouring and organs at risk (OARs) (such as lungs, heart, esophagus, spinal canal and contralateral breast) contouring were performed in accordance with the Varian Eclipse planning system (version 13.7 - Varian Medical Systems, Palo Alto, USA) in accordance with the RTOG atlas. [11] A breast and/or chest wall, and/or lymph node RT of 50 Gy in 25 fractions was planned for all patients, and an additional boost of $10 \mathrm{~Gy}$ in five fractions was planned for the patients requiring an RT boost. The 3DCRT, Field in Field (FIF), IMRT, and VMAT techniques were used in the treatment plans. The CTV coverage was required to be between $90 \%$ and $110 \%$ of the prescribed dose.

The Anisotropic Analytical Algorithm (AAA) photon dose calculation algorithm was used for all plans. The maximum dose rate was $400 \mathrm{MU} / \mathrm{min}$ for the IMRT and 3DCRT plans, and $600 \mathrm{MU} / \mathrm{min}$ for the VMAT plans. The dose calculation grid was selected to be $0.25 \mathrm{~cm}$. Treatment was delivered using a Varian linear accelerator (Clinac IX, Varian Medical Systems, Palo Alto, CA, USA).

The normality of the data was evaluated using a Shapiro-Wilk or Kolmogorov-Smirnov test, depending on the sample size. Since the data did not meet the normality criteria, it was summarized as median, minimum and maximum values. A Mann-Whitney $\mathrm{U}$ test was used for between-group comparisons, and a Kruskal-Wallis test was used for the comparison of more than two groups. After the Kruskal-Wallis test, pairwise comparisons were performed using the Conover method. The significance level was set at 0.05 for all analyses.

\section{Results}

The mean age of the patient group was 49 (range: 31-86) years; three had ductal carcinoma in situ while the others had invasive breast cancer; one patient was male; 12 patients were at stage $1 ; 39$ patients were at stage 2; 47 patients were at stage 3 and two patients 
Table 1. Patient characteristics

\section{Characteristics}

n (102)

Age

T stage

Tis (Carcinoma in situ)

$\mathrm{T} 1$

$\mathrm{T} 2$

T3

$\mathrm{T} 4$

$\mathrm{N}$ stage

NO

N1

N2

N3

Stage

0

I A

II $A$

II $B$

III $A$

III B

III C

IV

Pathology type

Invasive ductal carcinoma

Invasive lobular carcinoma

Medullary carcinoma

Mucinous carcinoma

Metaplastic carcinoma

Invasive carcinoma of no special type

Ductal carcinoma in situ (DCIS)

49 (Range:31-86)

mor side

Right

Left

Surgery

Mastectomy

Breast-conserving surgery

Axillary dissection

Yes

No

Neoadjuvant chemotherapy

Yes

No

Molecular subtypes

Luminal A

Luminal B

Triple-negative/basal-like

HER2-enriched

had oligometastatic cancer at the time of diagnosis. The metastatic patients underwent surgery followed by adjuvant RT once their metastases had disappeared following neoadjuvant chemotherapy.
Table 1. Cont.

\section{Characteristics}

n (102)

$\begin{array}{lr}\text { Estrogen receptor status } & \\ \quad \text { Positive } & 78 \\ \text { Negative } & 24 \\ \text { Progesterone receptor status } & \\ \text { Positive } & 67 \\ \text { Negative } & 35 \\ \text { Her-2 } & \\ \text { Positive } & 27 \\ \text { Negative } & 75\end{array}$

A total dose of 50 Gy was administered to 57 patients, and a boost treatment was administered to 46 patients as an additional 10 Gy in five fractions. The 3DCRT-FIF, IMRT and VMAT plans were delivered to 82, 3 and 17 patients, respectively. The mean CTV-50Gy volume was found to be $859 \mathrm{cc}$. The treatment and CTV parameter characteristics are specified in Table 2.

\begin{tabular}{|c|c|}
\hline \multicolumn{2}{|l|}{ CTV mastectomy } \\
\hline Volume (cc) & 859.8 \\
\hline Dmax (Gy) & 55.3 \\
\hline Dmean (Gy) & 50.9 \\
\hline V95 & 97 \\
\hline V100 & 70.2 \\
\hline V105 & 17.9 \\
\hline \multicolumn{2}{|l|}{ Radiotherapy volume } \\
\hline Breast & 25 \\
\hline Breast/chest wall+SCV+Ax & 19 \\
\hline Breast/chest wall+SCV+Ax+IMC LN & 58 \\
\hline \multicolumn{2}{|l|}{ Radiotherapy technique } \\
\hline FIF & 82 \\
\hline IMRT & 3 \\
\hline VMAT & 17 \\
\hline \multicolumn{2}{|l|}{ Boost } \\
\hline Yes & 45 \\
\hline No & 57 \\
\hline \multicolumn{2}{|l|}{ CTV boost } \\
\hline Volume (cc) & 86.86 \\
\hline \multicolumn{2}{|l|}{ Dmean (Gy) } \\
\hline V95 & 99.4 \\
\hline V100 & 90.4 \\
\hline V105 & 23.07 \\
\hline \multicolumn{2}{|l|}{ Boost technique } \\
\hline FIF & 17 \\
\hline IMRT & 7 \\
\hline VMAT & 3 \\
\hline Electron & 18 \\
\hline
\end{tabular}

FIF: Field in field; IMRT: Intensity-modulated radiation therapy; VMAT: Volumetric modulated arc therapy 
The mean lung volume was $4373 \mathrm{cc}$. The administered ipsilateral lung V20 and OAR doses are presented in Table 3. In the group receiving supraclavicular fossa RT, the mean V20Gy was found to be $24.8 \%$, compared to $16 \%$ in the group not receiving supraclavicular fossa RT $(\mathrm{p}<0.01)$. In the group receiving mammaria interna (MI) RT, the mean ipsilateral lung V20Gy was found to be $26.2 \%$, compared to $18.6 \%$ in the group not treated with MI RT $(\mathrm{p}<0.01)$. In the group treated with MI RT, the mean heart dose was $3.1 \mathrm{~Gy}$, compared to $2.1 \mathrm{~Gy}$ in the group not given $\mathrm{MI}$ RT ( $\mathrm{p}=0.04$ ). The mean ipsilateral lung V20Gy dose was found to be $16.5 \%$ in 25 patients who underwent breast RT alone, and $24.6 \%$ in the patients who underwent additional chest wall RT. The heart Dmean was found to be $2.5 \mathrm{~Gy}$ in 11 patients with left breast cancer who only underwent breast RT.
The detailed comparison of the techniques and the associated OAR doses is presented in Table 4.

\section{Discussion}

Breast cancer can be detected at an early stage. The frequency of treatment with RT following breast-conserving surgery has increased in recent years. There have been many young breast cancer patients with long survival expectancy, and so the amount of radiation received by healthy tissue becomes significant, [12] and this has led to the development of different respiratory management strategies in recent years.[13] For instance, ABC DIBH uses a spirometer-like device,[14] while Percussion-Assisted Radiation Therapy (PART) aims to suppress respiratory motion, while maintaining adequate oxygen diffusion and carbon dioxide removal

Table 3. OAR doses

\begin{tabular}{|c|c|c|c|c|}
\hline & \multicolumn{2}{|c|}{ Left } & \multicolumn{2}{|c|}{ Right } \\
\hline & Mastectomy $(n=34)$ & $B C B^{*}(n=26)$ & Mastectomy $(n=23)$ & BCS* $^{*}(n=19)$ \\
\hline \multicolumn{5}{|l|}{ Right lung } \\
\hline V5 & $8.1(0-32.7)$ & $2.3(0-38)$ & $48(39-74)$ & $39.9(23-61)$ \\
\hline V10 & $1.3(0-7.7)$ & $0.5(0-8)$ & $33.5(28-47)$ & $25.7(15-40)$ \\
\hline V20 & $0.05(0-0.9)$ & $0.02(0-0.08)$ & $25.3(16-35)$ & $17.7(11-29)$ \\
\hline Dmean (Gy) & $1.4(0-4.5)$ & $0.67(0-5)$ & $13.6(10-18)$ & $10.9(7-16)$ \\
\hline \multicolumn{5}{|l|}{ Left lung } \\
\hline V5 & $54(34-76.5)$ & $46.7(23-69)$ & $2.2(0-35)$ & 0.01 \\
\hline V10 & $36.6(19-53)$ & $30.4(12-44)$ & $0.43(0-6)$ & 0 \\
\hline V20 & $24.3(12-35)$ & $21.7(7-34)$ & 0 & 0 \\
\hline Dmean (Gy) & 13.5 (7.94-18.29) & $12.6(6-18)$ & $0.41(0-5)$ & $0.33(0-1)$ \\
\hline \multicolumn{5}{|l|}{ Heart } \\
\hline Dmax (Gy) & $39.5(4.2-55.3)$ & $36.4(6-56)$ & $12.9(4-51)$ & $9.2(3-29)$ \\
\hline Dmean (Gy) & $4(0.6-17.6)$ & $2.9(0-8)$ & $1.08(0-6)$ & $1.03(0-3)$ \\
\hline V20 & $2.3(0-9.7)$ & $2.13(0-12)$ & $0.12(0-2)$ & 0 \\
\hline V25 & $1.7(0-6.68)$ & $1.6(0-11)$ & $0.09(0-2)$ & 0 \\
\hline V30 & $1.2(0-5.9)$ & $1.4(0-10)$ & $0.07(0-2)$ & 0 \\
\hline V40 & $0.6(0-4.2)$ & $0.92(0-9)$ & $0.05(0-1)$ & 0 \\
\hline \multicolumn{5}{|l|}{ Esophagus } \\
\hline Dmax (Gy) & $38.2(7.1-52.6)$ & $24.2(1-48)$ & $32.6(8-49)$ & $8.3(1-50)$ \\
\hline \multicolumn{5}{|c|}{ Contralateral breast } \\
\hline Dmax (Gy) & $9.7(1.6-35.7)$ & $7.3(2-26)$ & $8.25(2-40)$ & $5.2(1-21)$ \\
\hline Dmean (Gy) & $1.1(0.04-3.2)$ & $0.37(0-3)$ & $0.29(0-3)$ & $0.3(0-1)$ \\
\hline \multicolumn{5}{|l|}{ Spinal canal } \\
\hline Dmax (Gy) & $10.6(0.4-29.95)$ & $6.67(1-37)$ & $11.2(2-36)$ & $3.02(0-20)$ \\
\hline \multicolumn{5}{|l|}{ Liver } \\
\hline Dmean (Gy) & $0.4(0-3.9)$ & 0 & $2.38(0-8)$ & $1.3(0-7)$ \\
\hline V20 & $0.1(0-4.6)$ & 0 & $3.3(0-15)$ & $1.2(0-12)$ \\
\hline
\end{tabular}

BCS*: Breast-conserving surgery 
Table 4. OAR doses according to treatment techniques

\begin{tabular}{|c|c|c|c|c|}
\hline Parameters & FIF-3DCRT & IMRT & VMAT & $\mathbf{p}$ \\
\hline \multicolumn{5}{|c|}{ Ipslateral lung } \\
\hline Dmean & $12.9(6.7-18.29)$ & $14.2(11.9-14.39)$ & $13.5(5.6-18.1)$ & 0.7328 \\
\hline V5 & $45.65(23.4-63.8)^{a}$ & $73.4(57.3-76.5)^{b}$ & $63.5(23-73.5)^{\mathrm{b}}$ & $<0.001$ \\
\hline V10 & $30.9(14.5-44)^{\mathrm{a}}$ & $46.3(34.7-49.5)^{\mathrm{b}}$ & $40.3(11.8-53.1)^{\mathrm{b}}$ & $<0.001$ \\
\hline V20 & $22.85(10.8-35.7)$ & $23.7(18.1-25.4)$ & $22.3(6.9-33.5)$ & 0.997 \\
\hline \multicolumn{5}{|c|}{ Kontralaterallung } \\
\hline Dmean & $0.2(0.1-3.3)^{\mathrm{a}}$ & $1.9(1.8-3)^{\mathrm{b}}$ & $3.78(0.2-4.9)^{b}$ & $<0.001$ \\
\hline V5 & $0(0-20.1)^{a}$ & $10.5(5.5-25.8)^{\mathrm{a}}$ & $21.1(0-37.9)^{\mathrm{b}}$ & $<0.001$ \\
\hline V10 & $0(0-4.3)^{a}$ & $0.2(0-1.2)^{b}$ & $3.7(0-7.9)^{b}$ & $<0.001$ \\
\hline V20 & $0(0-0)^{a}$ & $0(0-0)^{\mathrm{a}}$ & $0.06(0-0.9)^{\mathrm{b}}$ & $<0.001$ \\
\hline \multicolumn{5}{|l|}{ Heart } \\
\hline Dmean & $1.35(0.19-17.61)^{a}$ & $6.1(4.5-9.1)^{\mathrm{b}}$ & $4.5(0.2-6.8)^{\mathrm{b}}$ & $<0.001$ \\
\hline Dmax & $17.84(3.36-56.07)$ & $49.2(38.6-49.6)$ & $31.6(6.4-55.3)$ & 0.0965 \\
\hline V20 & $0(0-12)^{a}$ & $6.6(2.4-9.7)^{b}$ & $0.9(0-5.9)^{\mathrm{b}}$ & 0.0035 \\
\hline V25 & $0(0-11)^{a}$ & $4.2(1.1-6.4)^{b}$ & $0.01(0-3.8) a$ & 0.0242 \\
\hline V30 & $0(0-10.3)^{a}$ & $2.7(0.6-3.8)^{b}$ & $0(0-2.24)^{a}$ & 0.036 \\
\hline V40 & $0(0-8.5)$ & $0.4(0-1)$ & $0(0-1.1)$ & 0.4342 \\
\hline \multicolumn{5}{|l|}{ Esophagus } \\
\hline Dmax & $28.48(0.5-49.8)^{\mathrm{a}}$ & $51.3(46.3-52.6)^{b}$ & $39.8(2-50.7)^{b}$ & 0.002 \\
\hline \multicolumn{5}{|c|}{ Contralateral breast } \\
\hline Dmax & $3.8(1.2-40)^{a}$ & $13(5.4-22.9)^{b}$ & $15(5.2-35.7)^{b}$ & $<0.001$ \\
\hline Dmean & $0.18(0-2.3)^{a}$ & $3.2(0.6-16.6)^{b}$ & $2.3(0.04-2.96)^{b}$ & $<0.001$ \\
\hline \multicolumn{5}{|l|}{ Liver } \\
\hline Dmean & $0.25(0-8.09)$ & $0(0-0.7)$ & $0(0-7.4)$ & 0.2845 \\
\hline V20 & $0(0-14.9)$ & $0(0-0)$ & $0(0-9.8)$ & 0.095 \\
\hline \multicolumn{5}{|l|}{ Spinal canal } \\
\hline Max & $3.28(0.4-36.7)^{\mathrm{a}}$ & $17.1(11.3-29.95)^{b}$ & $16.66(1.9-35.9)^{b}$ & $<0.001$ \\
\hline
\end{tabular}

*a and b values indicate statistically significant treatment methods. FIF-3DCRT: Field in Field-threedimensional conformal radiation therapy; IMRT: Intensitymodulated radiation therapy; VMAT: Volumetric modulated arc therapy.

without general anesthesia.[15] It has been shown that $\mathrm{ABC}$ DIBH could reduce doses in the bilateral lungs, heart, left anterior descending coronary artery, contralateral breast, esophagus and spinal canal.[14]Continuous positive airway pressure (CPAP) significantly reduces tumor motion and increases total lung volume, thus contributing to reduced radiation doses to the lung and heart for lung stereotactic Body Radiation. [16] The CPAP technique has also been found to reduce ipsilateral lung and heart dosage in breast cancer RT through the use of conventional tangential fields. [17] Another respiratory motion technique uses rapid swallow non-invasive ventilation to regularize and to minimize the target variations resulting from respiratory motion.[18] The present study evaluates the clinical and dosimetric results of the application of the vDIBH technique in our clinic.

The doses absorbed by the lungs have been shown to be less with DIBH when compared to free-breathing in many studies,[19,20] although Bolukbaşı et al.[21] found no significant dose reduction in the lung, while V20 and V10 doses for the heart were reduced with the help of DIBH.In the present study, ipsilateral lung doses were found to be higher than in literature, which may be attributed to the fact that our patients were not homogeneous, that most had advanced stages of the disease and that boost doses were also considered in the relevant patients.

Supraclavicular region RT increases the doses absorbed by the lungs. In the present study, the lung Dmean, V5, V10 and V20 values were statistically higher in the patients who underwent additional supraclavicular RT, and these values are consistent with the literature.[9]

The risk of cardiovascular mortality has been shown to increase after breast and/or chest wall RT.[4,22] A $30 \%$ increased risk of mortality due to heart disease was identified in the Early Breast Cancer Trialists' Col- 
laborative Group study.[2] Each 1 Gy increase in the mean heart dose leads to $4-7 \%$ increased risk of heart disease and coronary events. $[23,24]$ The International Quantitative Analysis of Normal Tissue Effects in the Clinic (QUANTEC) guidelines state that for partial heart irradiation, "V25Gy $<10 \%$ will be associated with a $1 \%$ probability of cardiac mortality" in long-term follow-up after RT.[23] In the present study, the mean V25 value was found to be quite low, while Dmean, V10 and V20 values were compatible with the literature.[9,25]

Regional nodal irradiation increases the doses absorbed by the lungs and heart, with IMC irradiation in particular increasing heart doses compared to breast and chest wall irradiation alone.[26] In the present study, lung Dmean, V5, V10 and V20 doses, heart Dmean and V20 doses, as well as Dmax doses of the esophagus, spinal canal, and contralateral breast were found to be high in the group who underwent additional IMC-RT.

In line with our constitutional guidelines, the FIF technique was first considered for all breast cancer patients, while IMRT and VMAT techniques were applied when the appropriate doses could not be achieved by the FIF technique. Our institute criterion for ipsilateral lung V20Gy is $35 \%$. For the patients that FIF technique could not provide this criterion VMAT or IMRT was chosen for the treatment. The patients who were treated with the IMRT and VMAT techniques were generally patients who had additional regional lymph node RT, particularly IMC-RT. Since the IMRT technique was applied only to three patients, and the VMAT technique was applied to 17 patients, the comparison was not optimal, although contralateral lung doses, contralateral breast doses, spinal canal doses and esophagus doses were higher with these two techniques compared to FIF.

The limitations of this study include: All patients had RPM, and so there was no patient group without RPM. Furthermore, the number of patients was not homogeneous for different techniques.

\section{Conclusion}

Our retrospective study showed that the vDIBH technique could reduce ipsilateral lung and heart doses in accordance with the literature. Besides, we showed that for the patients that FIF technique could not be applied due to unsatisfied OAR dose restrictions, VMAT and IMRT are feasible alternatives. Patient compliance, however, is crucial. As the method increases the duration of treatment, it can be considered an applicable and effective treatment method only if appropriate patients are selected.

Peer-review: Externally peer-reviewed.

\section{Conflict of Interest: None.}

Ethics Committee Approval: Yes.

\section{Financial Support: None.}

Authorship contributions: Concept - O.T., M.D.; Design - S.G.; Supervision - E.K.P., B.D.; H.G. - None; Materials - M.D.; Data collection and/or processing - O.T.; Data analysis and/or interpretation - F.A.E.; Literature search - M.D.; Writing - O.T., B.T.U.; Critical review - O.T.

\section{References}

1. Bray F, Ferlay J, Soerjomataram I, Siegel RL, Torre LA, Jemal A. Global cancer statistics 2018: GLOBOCAN estimates of incidence and mortality worldwide for 36 cancers in 185 countries. CA Cancer J Clin 2018;68(6):394-424.

2. Early Breast Cancer Trialists' Collaborative Group (EBCTCG), Darby S, McGale P, Correa C, Taylor C, Arriagada R, et al. Effect of radiotherapy after breast-conserving surgery on 10-year recurrence and 15-year breast cancer death: meta-analysis of individual patient data for 10,801 women in 17 randomised trials. Lancet 2011;378(9804):1707-16.

3. Darby SC, McGale P, Taylor CW, Peto R. Long-term mortality from heart disease and lung cancer after radiotherapy for early breast cancer: prospective cohort study of about 300,000 women in US SEER cancer registries. Lancet Oncol 2005;6(8):557-65.

4. Cuzick J, Stewart H, Rutqvist L, Houghton J, Edwards R, Redmond C, et al. Cause-specific mortality in long-term survivors of breast cancer who participated in trials of radiotherapy. J Clin Oncol 1994;12(3):447-53.

5. Aznar MC, Duane FK, Darby SC, Wang Z, Taylor CW. Exposure of the lungs in breast cancer radiotherapy: $\mathrm{A}$ systematic review of lung doses published 2010-2015. Radiother Oncol 2018;126(1):148-54.

6. Bartlett FR, Colgan RM, Carr K, Donovan EM, McNair HA, Locke I, et al. The UK HeartSpare Study: randomised evaluation of voluntary deep-inspiratory breath-hold in women undergoing breast radiotherapy. Radiother Oncol 2013;108(2):242-7.

7. Bergom C, Currey A, Desai N, Tai A, Strauss JB. Deep Inspiration Breath Hold: Techniques and Advantages for Cardiac Sparing During Breast Cancer Irradiation. Front Oncol 2018;8:87.

8. Swanson T, Grills IS, Ye H, Entwistle A, Teahan M, 
Letts $\mathrm{N}$, et al. Six-year experience routinely using moderate deep inspiration breath-hold for the reduction of cardiac dose in left-sided breast irradiation for patients with early-stage or locally advanced breast cancer. Am J Clin Oncol 2013;36(1):24-30.

9. Nissen HD, Appelt AL. Improved heart, lung and target dose with deep inspiration breath hold in a large clinical series of breast cancer patients. Radiother Oncol 2013;106(1):28-32.

10. Mohamad O, Shiao J, Zhao B, Roach K, Ramirez E, Vo DT, et al. Deep inspiration breathhold for left-sided breast cancer patients with unfavorable cardiac anatomy requiring internal mammary nodal irradiation. Pract Radiat Oncol 2017;7(6):361-7.

11.NRG. Breast Cancer Atlas. Available at:https:// www.nrgoncology.org/Portals/0/Scientific $\% 20$ Program/CIRO/Atlases/BreastCancerAtlas_corr. pdf?ver=2018-04-18-144201-270 Accessed Sep 3, 2020.

12. Sager O, Beyzadeoglu M, Dincoglan F, Oysul K, Kahya Y.E, Gamsiz H. The Role of Active Breathing Control-Moderate Deep Inspiration Breath-Hold $(\mathrm{ABC}-\mathrm{mDIBH})$ Usage in non-Mastectomized Leftsided Breast Cancer Radiotherapy: A Dosimetric Evaluation. UHOD.2019, Vol 29, Num 4 Page(s): 147-155.

13. Remouchamps VM, Vicini FA, Sharpe MB, Kestin LL, Martinez AA, Wong JW. Significant reductions in heart and lung doses using deep inspiration breath hold with active breathing control and intensity-modulated radiation therapy for patients treated with locoregional breast irradiation. Int J Radiat Oncol Biol Phys 2003;55(2):392-406.

14. Dincoglan F, Beyzadeoglu M, Sager O, Oysul K, Kahya YE, Gamsiz H, Uysal B, Demiral S, Dirican B, Surenkok S. Dosimetric evaluation of critical organs at risk in mastectomized left-sided breast cancer radiotherapy using breath-hold technique. Tumori. 2013 JanFeb;99(1):76-82. doi: 10.1700/1248.13792.

15. Péguret N, Ozsahin $M$, Zeverino $M$, Belmondo B, Durham AD, Lovis A, et al. Apnea-like suppression of respiratory motion: First evaluation in radiotherapy. Radiother Oncol 2016;118(2):220-6.

16. Goldstein JD, Lawrence YR, Appel S, Landau E, Ben-David MA, Rabin T, et al. Continuous Positive Airway Pressure for Motion Management in Stereotactic Body Radiation Therapy to the Lung: A Controlled Pilot Study. Int J Radiat Oncol Biol Phys 2015;93(2):391-9.

17. Kil WJ, Pham T, Kim K. Heart sparing breast cancer radiotherapy using continuous positive airway pres- sure (CPAP) and conventional supine tangential fields: an alternative method for patients with limited accessibility to advanced radiotherapy techniques. Acta Oncol 2019 Jan;58(1):105-9.

18. West NS, Parkes MJ, Snowden C, Prentis J, McKenna J, Iqbal MS, et al. Mitigating Respiratory Motion in Radiation Therapy: Rapid, Shallow, Non-invasive Mechanical Ventilation for Internal Thoracic Targets. Int J Radiat Oncol Biol Phys 2019;103(4):1004-10.

19. Chi F, Wu S, Zhou J, Li F, Sun J, Lin Q, et al. Dosimetric comparison of moderate deep inspiration breathhold and free-breathing intensity-modulated radiotherapy for left-sided breast cancer. Cancer Radiother 2015;19(3):180-6.

20. Rice L, Goldsmith C, Green MM, Cleator S, Price PM. An effective deep-inspiration breath-hold radiotherapy technique for left-breast cancer: impact of post-mastectomy treatment, nodal coverage, and dose schedule on organs at risk. Breast Cancer (Dove Med Press) 2017;9:437-46.

21. Bolukbasi Y, Saglam Y, Selek U, Topkan E, Kataria A, Unal Z, Alpan V. Reproducible deep-inspiration breath-hold irradiation with forward intensity-modulated radiotherapy for left-sided breast cancer significantly reduces cardiac radiation exposure compared to inverse intensity-modulated radiotherapy. Tumori 2014;100(2):169-78.

22. Taylor C, Correa C, Duane FK, Aznar MC, Anderson SJ, Bergh J, et al. Estimating the Risks of Breast Cancer Radiotherapy: Evidence From Modern Radiation Doses to the Lungs and Heart and From Previous Randomized Trials. J Clin Oncol 2017;35(15):1641-9.

23. Darby SC, Ewertz M, McGale P, Bennet AM, Blom-Goldman U, Brønnum D, et al. Risk of ischemic heart disease in women after radiotherapy for breast cancer. N Engl J Med 2013;368(11):987-98.

24. Sardaro A, Petruzzelli MF, D’Errico MP, Grimaldi L, Pili G, Portaluri M. Radiation-induced cardiac damage in early left breast cancer patients: risk factors, biological mechanisms, radiobiology, and dosimetric constraints. Radiother Oncol 2012;103(2):133-42.

25. Wiant D, Wentworth S, Liu H, Sintay B. How Important Is a Reproducible Breath Hold for Deep Inspiration Breath Hold Breast Radiation Therapy? Int J Radiat Oncol Biol Phys. 2015 Nov 15;93(4):901-7.

26. Chargari C, Castadot P, Macdermed D, Vandekerkhove C, Bourgois N, Van Houtte P, et al. Internal mammary lymph node irradiation contributes to heart dose in breast cancer. Med Dosim 2010;35(3):163-8. 